\title{
The polytrauma clinical triad in patients with chronic pain after motor vehicle collision
}

This article was published in the following Dove Press journal: Journal of Pain Research

\author{
Cayden Peixoto' \\ Lindsay Hyland ${ }^{2}$ \\ Derrick Matthew Buchanan² \\ Erika Langille ${ }^{2}$ \\ Richard Nahas ${ }^{3}$ \\ 'The Seekers Centre, Ottawa, \\ ON, Canada; ${ }^{2}$ Department of \\ Neuroscience, Carleton University, \\ Ottawa, ON, Canada; ${ }^{3}$ Department of \\ Family Medicine, University of Ottawa, \\ Ottawa, ON, Canada
}

Correspondence: Cayden Peixoto

The Seekers Centre, 942 Merivale Road, Ottawa, ON KIZ 5Z9, Canada

Tel +6137277246

Fax +6I3 7277247

Email cayden@seekerscentre.com
Background: The polytrauma clinical triad (PCT) is a complex disorder composed of three comorbid diagnoses of chronic pain, post-traumatic stress disorder (PTSD), and postconcussion syndrome (PCS). PCT has been documented in veterans returning from deployment, but this is the first report on PCT prevalence in nonmilitary personnel after a motor vehicle collision (MVC). Methods: Data were drawn from routine intake assessments completed by 71 patients referred to a community-based clinic for chronic pain management. All patients completed the posttraumatic stress disorder checklist for the Diagnostic and Statistical Manual of Mental Disorders, fifth edition (PCL-5), and Rivermead Post-Concussion Symptoms Questionnaire (RPQ) during a standardized intake assessment. An additional modified RPQ score was derived to address previously reported symptom overlap between PCS and chronic pain.

Results: Standard and modified RPQ scores yielded PCS prevalence rates of $100 \%$ and $54.9 \%$ in our sample, respectively. Results suggest that a modified RPQ score, limited to visual and vestibular symptoms, may be more useful PCS screening criteria in patients with chronic pain. PTSD screening criteria on the PCL-5 were met by $85.9 \%$ of the patients. More than half of the patients referred for chronic pain after MVC met criteria for PCT (52.1\%). Patients who met PCT criteria reported worse headache, overall pain, and sleep quality outcomes.

Conclusion: Among patients in our sample with chronic pain after MVC, more than half met criteria for PCT. A modified approach to RPQ scoring limited to visual and vestibular symptoms may be required to screen for PCS in these patients.

Keywords: chronic pain, mTBI, concussion, PTSD, MVC

\section{Introduction}

Recent reports have identified clusters of comorbid symptoms among military personnel with chronic postdeployment health complaints. ${ }^{1}$ One such cluster has been called the polytrauma clinical triad (PCT), and includes chronic pain, post-traumatic stress disorder (PTSD), and postconcussion syndrome (PCS). ${ }^{2,3}$

While PCT has been reported in military personnel, it has not been described in other populations. Many of the risk factors for developing concurrent chronic pain, PTSD, and PCS are also present in individuals who experience motor vehicle collisions (MVCs). These include immediate onset of pain, emotional trauma, and sudden changes in the velocity of the brain that can result in mild traumatic brain injury (mTBI). ${ }^{2,4}$ While traumatic injuries in military personnel are caused by blasts or bullets, ${ }^{2}$ a common cause in civilians is MVC. ${ }^{5,6}$ These can be classified as polytrauma injuries, defined by the US Department of Veteran Affairs as "two or more injuries to physical regions or 
organ systems, one of which may be life threatening, resulting in physical, cognitive, psychological, or psychosocial impairments and functional disability". ${ }^{7}$

We propose that the mechanism of injury in MVC incorporates many of the risk factors implicated in combatrelated polytrauma injuries. It is well documented that brain injury ${ }^{8-10}$ and PTSD ${ }^{11,12}$ commonly occur after MVC, and that chronic pain syndromes commonly develop after whiplash injuries. ${ }^{13,14}$ These MVC-related disorders are believed to occur as a result of many of the same risk factors as those that can complicate blast injuries and other injuries that occur during combat. Pain can occur as a result of direct trauma to ligaments and tendons, with associated muscle spasm and cartilage injury that cannot be diagnosed by conventional diagnostic imaging. Emotional trauma can occur as a result of the shock of the impact, the potential threat to life, and the expected negative impact on one's future health, and personal and financial security. ${ }^{15,16}$ Our current understanding of the mechanism of traumatic brain injury makes it clear that diffuse axonal injury can occur from rapid deceleration with or without the direct impact that is recognized as a head injury. ${ }^{10,17}$ The combined impact of these three risk factors may create a "perfect storm" that can lead to PCT after MVC.

This study was conducted to estimate the prevalence of PCT in patients with chronic pain after MVC. This was suspected based on the high frequency of PCS and PTSD symptoms reported during standardized intake assessments. Based on the high prevalence of certain PCS symptoms in patients with chronic pain, ${ }^{18,19}$ we explored the potential value of placing more emphasis on visual and vestibular symptoms, which appear to be more specific to PCS.

\section{Methods}

We conducted an analysis of routinely collected data during screening appointments of patients referred to an MVC chronic pain management program at The Seekers Center, a community-based pain management clinic in Ottawa, Canada. From May 2016 to January 2017, a total of 95 patients underwent intake assessments to determine their eligibility, and to establish treatment priorities. These included the presence of PCS, PTSD, and other mood disorders.

Screening data were collected using the Ocean mobile data platform (CognisantMD, Toronto, ON, Canada). Information was entered on a tablet PC (Samsung) and synchronized with the clinic's electronic medical records software (OSCAR, McMaster University, Hamilton, ON, Canada). Each assessment lasted approximately 1 hour and was conducted in person at the clinic. Intakes relied on selfreported data using validated questionnaires completed with assistance from an experienced clinical associate. All patients were screened uniformly, and completed the full battery of questions and screening tools. An experienced pain physician reviewed all relevant clinical information during a brief patient interview at the end of each intake.

\section{Ethics}

This study was approved by the Bruyère Research Ethics Board. All patients provided written informed consent prior to data collection.

\section{Study population}

Prior to their intake assessment, patients referred to the clinic were triaged as acute (less than 3 months post-MVC; $n=24$ ), or chronic (greater than 3 months post-MVC; $n=71$ ). All patients were referred by physicians and other community providers. Chronic pain was defined as persistent pain in one or more body part lasting longer than 3 months. ${ }^{20}$ Only patients with chronic pain post-MVC $(n=71)$ were included in the study sample.

\section{Study measures \\ Pain}

All individuals in the sample were previously diagnosed with chronic pain, which was the reason for referral. Pain severity was estimated for headache pain and overall body pain using a visual analog scale from 0 ("no pain") to 10 ("worst pain imaginable"). Patients were asked to estimate their headache pain and body pain both during flares and at baseline (average pain experienced between flares) and to report their monthly average number of headaches.

During the intake assessment, patients also completed the painDETECT questionnaire, a validated screening tool for neuropathic pain, ${ }^{21}$ to further classify their pain as either nociceptive or neuropathic.

\section{Post-traumatic stress disorder}

The Post-traumatic Stress Disorder Checklist (PCL-5), a validated screening tool for PTSD and the official checklist of the Diagnostic and Statistical Manual of Mental Disorders, fifth edition, ${ }^{22}$ was used to estimate the prevalence of PTSD within the sample. The PCL-5 is a 20 -item questionnaire, with each item being scored on a 5-point Likert scale ranging from 0 ("Not at all") to 4 ("Extremely"). A total score of 44 suggests a high likelihood of PTSD diagnosis, ${ }^{23}$ and was used to identify PTSD in this study. 


\section{Postconcussion syndrome}

The Rivermead Post-Concussion Symptoms Questionnaire $(\mathrm{RPQ})^{24}$ was used to determine the presence of PCS in our sample. The RPQ is a 16-item questionnaire, with each item comparing a patient's pre- and post-collision state, and scored on a 5-point Likert scale ranging from 0 ("Not experienced at all") to 4 ("A severe problem"). We used a total score of $\geq 16$ on the standard 16-item RPQ to define PCS, as established by Thompson et al. ${ }^{25}$

We derived a modified PCS score because the validity of this questionnaire has been questioned in patients with chronic pain due to symptoms present in both disorders. ${ }^{18}$ This was consistent with our clinical experience, which has led us to place less emphasis on headaches, constitutional symptoms (sleep disturbance, fatigue, restlessness), mood symptoms (irritability, depression, frustration), or cognitive symptoms (poor memory, poor concentration, taking longer to think). Greater emphasis was placed on six questions related to vision (blurred vision, light sensitivity, double vision) and to hearing and vestibular function (dizziness, nausea, noise sensitivity). Previous studies have suggested that these symptoms are less severe and less frequently reported in isolated chronic pain syndromes, ${ }^{18,19}$ which is consistent with our clinical experience. We chose the name RPQ-6 to describe a modified score with values ranging from 0 to 24 . We defined PCS as a score of $\geq 12$ on the RPQ- 6 in this population with chronic pain.

\section{Demographic and MVC-related data}

Age and sex were documented as part of the screening intake. Patients also indicated whether or not they were driving at the time of collision, whether there were other passengers in the vehicle, the type of impact (front, rear, side, or other), whether they experienced a loss of consciousness, and whether they recalled any immediate symptoms of concussion (confusion, dizziness, nausea, tinnitus, blurry/double vision, or difficulty concentrating) on the scene.

\section{Emergency room medical care}

Patients were questioned about the medical care they received after their MVC. They were asked whether or not they visited a hospital emergency room within 24 hours of the collision. They were also asked whether brain-imaging studies were performed (magnetic resonance imaging $[\mathrm{MRI}]$ or computed tomography $[\mathrm{CT}]$ scan), whether medications were prescribed, and if they were given a diagnosis (concussion/mTBI and/or whiplash-associated disorder). Patient report was corroborated by medical record review whenever possible.

\section{Sleep quality}

Patients completed the Pittsburgh Sleep Quality Index (PSQI) during their intake assessment. The PSQI is a self-rated questionnaire that evaluates sleep quality and disturbances over the past month. ${ }^{26}$ It contains seven-component score items that assess overall sleep quality, latency, duration, efficiency, sleep disturbances, medication efficacy, and dysfunction while awake. These component items are scored on a 4-point Likert scale ranging from 0 to 3 . This corresponds to a total component score of $0-21$, where higher scores signify poorer sleep quality.

\section{PCT}

PCT prevalence in our sample was determined by chronic pain patients meeting screening criteria for both PTSD and PCS. Based on screening criteria results, patients with and without PCT were sorted into respective groups for comparative analysis.

\section{Data analysis}

Our data were exported from the Ocean database platform to Microsoft Excel for Mac 2011 Version 14.0.0, and analyzed using SPSS Statistics Version 20.0. Shapiro-Wilk tests were performed to test data for normality. Mann-Whitney U-tests were conducted in order to investigate significant differences in health outcome measures between patients with and without PCT. Group differences in these values were computed using two-tailed hypothesis at a statistical significance threshold of alpha $=0.05$.

\section{Results}

Descriptive statistics of the sample are shown in Table 1. The mean age of the overall sample was 43.3 years $(\mathrm{SD}=12.7)$, and slightly more than half of the patients were female $(\mathrm{n}=40 ; 56.3 \%)$. The mean duration from MVC to screening evaluation date was 753.6 days $(\mathrm{SD}=653.3$ ), and $69.0 \%$ of the study sample were screened more than 12 months after their MVC. Most patients were driving the vehicle involved (71.8\%), and a rear impact was most common (47.9\%). While the majority recalled experiencing one or more symptoms of concussion immediately following their collision (76.1\%), only a minority recalled a loss of consciousness (18.0\%). Within 24 hours of their MVC, $74.6 \%(n=53)$ of the sample visited an emergency room, and nearly three-quarters of these patients (38 of 53) were given prescription analgesics. Upon receiving any medical care after MVC, whiplash-associated disorder was diagnosed in $40.8 \%$ of the sample, and $26.8 \%$ were told that they had likely sustained a concussion. 
Table I Descriptive statistics of the study population $(n=7 I)$ and a subset of the sample composed of MVC patients with PCT ( $n=37)$

\begin{tabular}{|c|c|c|c|c|}
\hline \multirow[t]{2}{*}{ Sample characteristics } & \multicolumn{2}{|c|}{ Study sample $(n=7 I)$} & \multicolumn{2}{|c|}{ PCT group $(n=37)$} \\
\hline & $\mathbf{N}$ & $\%$ & $\mathbf{N}$ & $\%$ \\
\hline \multirow[t]{2}{*}{$\overline{\text { Age }}$} & \multirow{2}{*}{\multicolumn{2}{|c|}{$\begin{array}{l}\text { Mean }=43.3 \text { years } \\
(\mathrm{SD}=12.7)\end{array}$}} & \multirow{2}{*}{\multicolumn{2}{|c|}{$\begin{array}{l}\text { Mean }=42.6 \text { years } \\
(S D=10.7)\end{array}$}} \\
\hline & & & & \\
\hline \multicolumn{5}{|l|}{ Gender } \\
\hline Female & 40 & 56.3 & 24 & 64.9 \\
\hline Male & 31 & 43.7 & 13 & 35.1 \\
\hline \multicolumn{5}{|l|}{ Duration from MVC to intake assessment } \\
\hline Less than 12 months & 22 & 31.0 & 13 & 35.1 \\
\hline Greater than 12 months & 49 & 69.0 & 24 & 64.9 \\
\hline \multicolumn{5}{|l|}{ Driving demographics } \\
\hline In the driver seat at time of collision & 51 & 71.8 & 27 & 73.0 \\
\hline Had other passengers in the car & 26 & 36.6 & 15 & 40.5 \\
\hline \multicolumn{5}{|l|}{ Type of collision } \\
\hline Front impact & 12 & 16.9 & 5 & 13.5 \\
\hline Rear impact & 34 & 47.9 & 16 & 43.2 \\
\hline Side impact & 13 & 18.3 & 7 & 18.9 \\
\hline Other ${ }^{\mathrm{a}}$ & 12 & 16.8 & 9 & 24.3 \\
\hline \multicolumn{5}{|l|}{ Immediate to collision } \\
\hline Experienced loss of consciousness & 13 & 18.3 & 9 & 24.3 \\
\hline Immediate symptoms of concussion ${ }^{\mathrm{b}}$ & 54 & 76.1 & 32 & 86.5 \\
\hline \multicolumn{5}{|l|}{ Post-MVC emergency room patient care } \\
\hline Visited ER within 24 hours of MVC & 53 & 74.6 & 25 & 67.6 \\
\hline Prescribed medications for pain by ER doctor & 38 & 53.5 & 22 & 59.5 \\
\hline Image of head at ER visit ${ }^{\mathrm{c}}$ & 22 & 31.0 & 11 & 29.7 \\
\hline Concussion diagnosis & 19 & 26.8 & 10 & 27.0 \\
\hline Whiplash-associated disorder diagnosis & 29 & 40.8 & 16 & 43.2 \\
\hline Current analgesic use & 56 & 78.8 & 29 & 74.4 \\
\hline Prescription opioids & 17 & 23.9 & 6 & 15.4 \\
\hline Over-the-counter analgesics only & 39 & 54.9 & 23 & 59.0 \\
\hline
\end{tabular}

Notes: aMVC involved patient being struck by a vehicle while being a pedestrian or cyclist. blmmediate symptoms of concussion include experiencing confusion, dizziness, nausea, tinnitus, blurry/double vision, or difficulty concentrating. Images of head include either computed tomography scan or magnetic resonance imaging. Includes aceteminophen and over-the-counter nonsteroidal anti-inflammatory drugs such as acetylsalicylic acid, ibuprofen, and naproxen.

Abbreviations: ER, emergency room; MVC, motor vehicle collision; PCT, polytrauma clinical triad.

RPQ item scoring results are illustrated in Table 2. High item scores (defined as a score of $\geq 3$ ) for measures relating to headaches, mood disturbances (irritability, depressed mood, restlessness, and frustration), cognitive disturbances (forgetfulness, poor concentration, and thought processing), and constitutional symptoms (fatigue, sleep quality, and restlessness) were present in almost all patients. In contrast, there were lower incidences of patients reporting high item scores on RPQ measures relating to visual disturbances (blurred, vision, light sensitivity, and double vision) and vestibular disturbances (dizziness, nausea/vomiting, and noise sensitivity). Mean scores were also lower for visual and vestibular symptoms (1.775 and 2.380, respectively) than those for headache (3.535), mood (3.146), cognitive (3.061), and constitutional (3.174) PCS symptoms.

The RPQ-6 score significantly reduced the presumed prevalence of PCS in our chronic pain sample, as shown in Table 3. Using screening criteria established by Thompson et al (2016), an RPQ cutoff score of $\geq 16,{ }^{15} 100.0 \%$ ( $n=71$ ) of the study sample would have met the criteria for PCS. A modified RPQ-6 score of $\geq 12$ yielded an estimated PCS prevalence of $54.9 \%(n=39)$. The RPQ-6 had a Cronbach's alpha of 0.80 in our sample, indicating good internal reliability.

Approximately half of the patients in our sample (52.1\%; $\mathrm{n}=37$ ) met criteria for PCT (chronic pain, PTSD, and PCS). Based on standard PCL-5 scores and our modified RPQ-6 score, $85.9 \%(\mathrm{n}=61)$ of the sample met criteria for PTSD, and $54.9 \%(n=39)$ met criteria for PCS (Figure 1). In our sample, 24 patients (33.8\%) met criteria for PTSD but not PCS. Conversely, there were only two patients with chronic pain $(2.8 \%)$ who met criteria for PCS but not PTSD.

Patients who met our criteria for PCT reported significantly worse symptoms, as shown in Table 4. Mann-Whitney $U$-test analysis of these measures found significant increases in the PCT group for mean rank number of headaches per month $(U=344.5 ; Z=2.558 ; P=0.010)$, intensity of headache 
Table 2 Results of the Rivermead Post-Concussion Symptoms Questionnaire $(n=7 \mathrm{I})$

\begin{tabular}{|c|c|c|c|c|c|c|c|}
\hline \multirow[t]{2}{*}{ Measures } & \multicolumn{5}{|c|}{ Item score frequency } & \multirow{2}{*}{$\begin{array}{l}\text { Mean item(s) } \\
\text { score }\end{array}$} & \multirow{2}{*}{$\begin{array}{l}\text { Prevalence of a high } \\
\text { item score }(\%)^{\mathrm{a}}\end{array}$} \\
\hline & $\overline{0}$ & I & 2 & 3 & 4 & & \\
\hline Headaches & 1 & 2 & 5 & 13 & 50 & 3.535 & 88.7 \\
\hline Cognitive disturbances & & & & & & 3.061 & \\
\hline Forgetfulness & 3 & 3 & 7 & 27 & 31 & 3.127 & 81.7 \\
\hline Poor concentration & I & 3 & 6 & 37 & 24 & 3.127 & 85.9 \\
\hline Thought processing & 2 & 5 & 10 & 33 & 21 & 2.930 & 76.1 \\
\hline Mood disturbances & & & & & & 3.146 & \\
\hline Irritability & 2 & 3 & 12 & 29 & 25 & 3.014 & 76.1 \\
\hline Depressed mood & 1 & 0 & 12 & 25 & 33 & 3.254 & 81.7 \\
\hline Frustration & 2 & 1 & 7 & 34 & 27 & 3.169 & 85.9 \\
\hline Constitutional symptoms & & & & & & 3.174 & \\
\hline Fatigue & 1 & 0 & I & 23 & 46 & 3.592 & 93.0 \\
\hline Sleep quality & 2 & 0 & 4 & 20 & 45 & 3.493 & 91.5 \\
\hline Restlessness & 6 & 8 & 19 & 25 & 13 & 2.437 & 53.5 \\
\hline Visual disturbances & & & & & & 1.775 & \\
\hline Blurred vision & 20 & 4 & 21 & 19 & 7 & 1.845 & 36.6 \\
\hline Light sensitivity & 11 & 3 & 15 & 23 & 19 & 2.507 & 59.2 \\
\hline Double vision & 41 & 10 & 4 & 13 & 3 & 0.972 & 22.5 \\
\hline Vestibular disturbances & & & & & & 2.380 & \\
\hline Dizziness & 10 & I & 20 & 23 & 17 & 2.507 & 56.3 \\
\hline Nausea and/or vomiting & 21 & 7 & 13 & 21 & 9 & 1.859 & 42.3 \\
\hline Noise sensitivity & 10 & 3 & 10 & 18 & 30 & 2.775 & 67.6 \\
\hline
\end{tabular}

Note: a Defined by patients reporting a score of $\geq 3$ on the corresponding Rivermead Post-Concussion Symptoms Questionnaire item measure.

Table 3 Comparison of PCS, PTSD, and PCT prevalence using different screening criteria for PCS in a sample of chronic pain patients after an MVC $(n=7 I)$

\begin{tabular}{|c|c|c|}
\hline Disorder measure & RPQ & RPQ-6 \\
\hline \multicolumn{3}{|l|}{ Postconcussion syndrome } \\
\hline Mean RPQ score (SD) & $44.14(10.45)$ & $12.47(5.78)$ \\
\hline Prevalence & $100.0 \%(n=7 \mid)^{a}$ & $54.9 \%(n=39)^{b}$ \\
\hline \multicolumn{3}{|l|}{ Post-traumatic stress disorder } \\
\hline Mean PCL-5 score (SD) & $56.90(14.65)$ & $56.90(14.65)$ \\
\hline Prevalence $^{c}$ & $85.9 \%(n=6 I)$ & $85.9 \%(n=61)$ \\
\hline \multicolumn{3}{|l|}{ Polytrauma clinical triad } \\
\hline Prevalence $^{d}$ & $85.9 \%(n=6 I)$ & $52.1 \%(n=37)$ \\
\hline
\end{tabular}

Notes: ${ }^{2}$ Defined by patients meeting screening criteria for PCS established by Thompson et a ${ }^{25}$ : a total score of $\geq 16$ on the Rivermead Post-Concussion Symptoms Questionnaire. ${ }^{14}$ 'Defined by patients meeting screening criteria for PCS proposed in this study: a total score of $\geq 12$ on the six items of the RPQ that relate to vestibular and visual symptoms of concussion. 'Defined by patients meeting screening criteria for PTSD: a total score of $\geq 44$ on the Post-traumatic Stress Disorder checklist. ${ }^{d}$ Based on chronic pain patients screening positive for both PTSD and PCS.

Abbreviations: MVC, motor vehicle collision; PCL-5, post-traumatic stress disorder checklist for the Diagnostic and Statistical Manual of Mental Disorders, fifth edition; PCS, postconcussion syndrome; PCT, polytrauma clinical triad; PTSD, posttraumatic stress disorder; RPQ-6, six-item Rivermead Post-Concussion Symptoms Questionnaire.

pain at baseline $(U=396 ; Z=2.676 ; P=0.007)$ and during flares $(U=230 ; Z=2.401 ; P=0.016)$, intensity of body pain at baseline ( $U=372.5 ; Z=2.947 ; P=0.003)$ and during flares ( $U=274 ; Z=2.244 ; P=0.025)$, and Pittsburgh Sleep Quality Index score $(U=430 ; Z=2.133 ; P=0.033)$.

\section{Discussion}

To our knowledge, this is the first report of the PCT in a civilian population. This syndrome, which includes symptoms consistent with chronic pain, PTSD, and PCS, was highly prevalent in our sample of patients referred for chronic pain after MVC. Using the PCL-5 and our modified version of the $\mathrm{RPQ}$, we found a $52.1 \%$ prevalence of PCT in this population.

The prevalence of PCT in our sample is greater than the $42.1 \%$ prevalence rate reported by Lew et al (2009) in a review of the medical records of 340 Operation Iraqi Freedom/Operation Enduring Freedom veterans seen at a Department of Veterans Affairs Polytrauma Network Site. ${ }^{2}$ However, our study population was limited to patients referred for chronic pain management, which may not directly translate to a normal sample. Notably, among military personnel with chronic pain in the aforementioned study ( $\mathrm{n}=277)$, PCT was present in $51.6 \%$, which is remarkably similar to our findings.

Interestingly, our sample presented with a lower incidence of PCS than PTSD. While these disorders were more commonly reported together, patients with chronic pain after MVC were more likely to have PTSD with no PCS, than they were to have PCS with no PTSD. One possible explanation is that while military personnel are psychologically prepared and trained for emotionally traumatic events, civilians are less prepared, having no formal training to help 


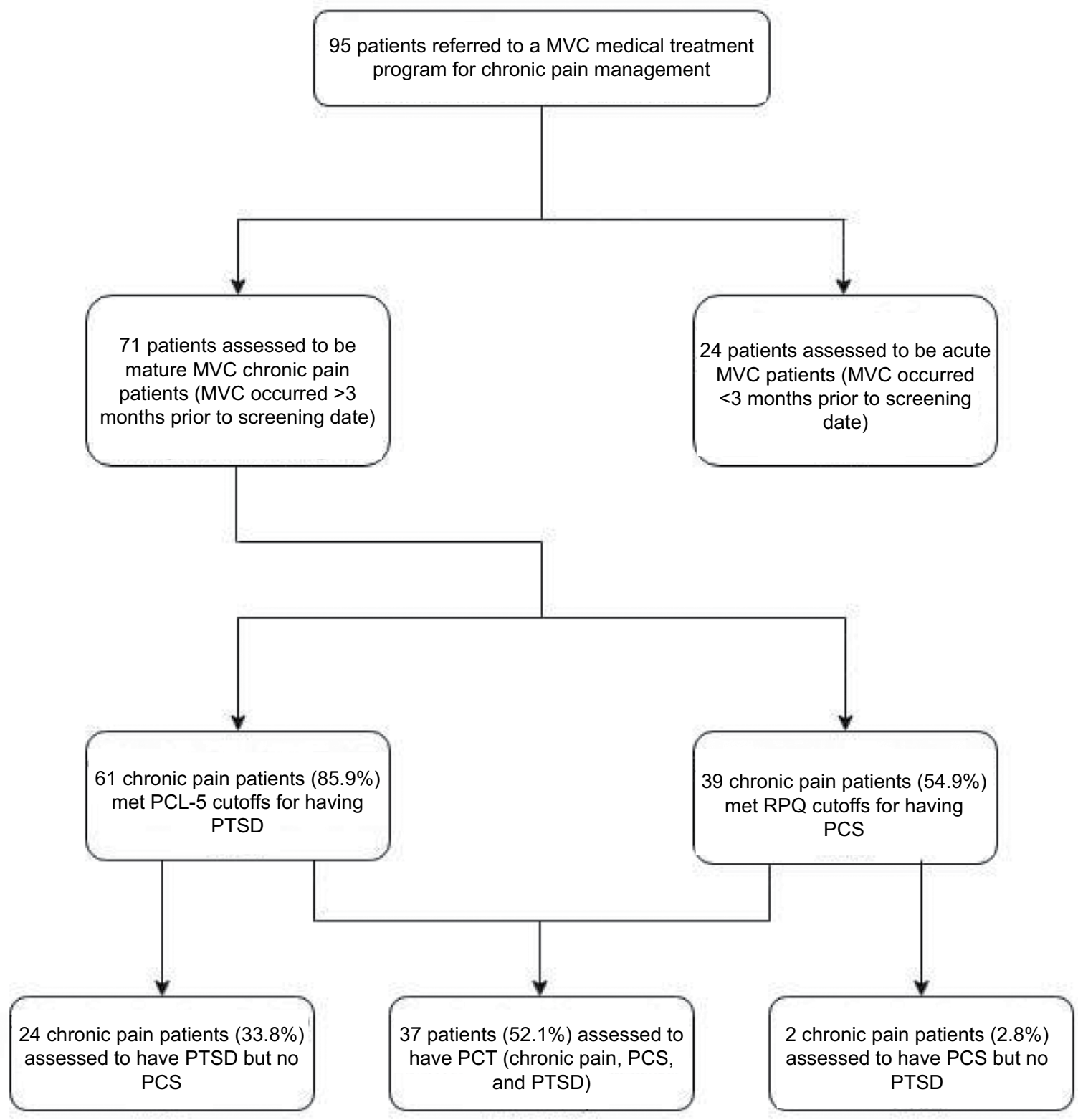

Figure I The polytrauma clinical triad after MVC.

Notes: Distribution of patients with chronic pain, PCS, and PTSD in a sample of patients referred to The Seekers Center for chronic pain management following an MVC. Abbreviations: MVC, motor vehicle collision; PCL-5, post-traumatic stress disorder checklist for the Diagnostic and Statistical Manual of Mental Disorders, fifth edition; PCS, postconcussion syndrome; PCT, polytrauma clinical triad; PTSD, post-traumatic stress disorder; RPQ, Rivermead Post-Concussion Symptoms Questionnaire.

them cope. Physical deconditioning, medicolegal stressors, a sense of injustice, and uncertain disability benefits may also be mitigating factors.

Our findings of comorbid PCS and PTSD are consistent with those of Lagarde et al (2014), who found mTBI to be correlated to PTSD development. ${ }^{27}$ They also support data reported by Brenner et al (2010), who suggest that PCS is more common in patients with PTSD after mTBI than in those with either mTBI or PTSD alone. ${ }^{28}$ There appears to be a complex interplay between PTSD and PCS, which may be linked to the limbic pathways that are shedding light on PTSD as an organic brain syndrome. ${ }^{29,30}$

Significantly worse pain, headache, and sleep quality outcomes were reported in patients with PCT in our sample. Pain can be aggravated by other unpleasant symptoms, including those seen in PTSD and PCS., ${ }^{2,31,32}$ This may partly explain the increased pain severity reported in our patients with PCT.

While differences in painDetect scores were not statistically significant between patients with and without PCT, there was a trend toward an increased risk of neuropathic pain 
Table 4 Independent samples, two-tailed Mann-Whitney U-test comparisons of headache, sleep quality, and pain outcomes in motor vehicle collision patients with and without PCT

\begin{tabular}{|c|c|c|c|c|c|}
\hline \multirow[t]{2}{*}{ Health outcomes } & \multicolumn{2}{|c|}{$\begin{array}{l}\text { PCT sample } \\
(n=37)\end{array}$} & \multicolumn{2}{|c|}{$\begin{array}{l}\text { Non-PCT } \\
\text { sample } \\
(n=34)\end{array}$} & \multirow[t]{2}{*}{$P$-value } \\
\hline & Mean & SD & Mean & SD & \\
\hline \multicolumn{6}{|l|}{ Headaches } \\
\hline Headaches per month & 18.94 & 10.10 & 12.27 & 10.04 & 0.010 \\
\hline Baseline headache pain & 7.24 & 1.80 & 5.41 & 3.01 & 0.007 \\
\hline Headache flare-up pain & 9.48 & 0.68 & 8.42 & 1.69 & 0.016 \\
\hline \multicolumn{6}{|l|}{ Sleep quality } \\
\hline $\begin{array}{l}\text { Pittsburgh Sleep Quality } \\
\text { Index Score }\end{array}$ & 17.17 & 5.50 & 14.20 & 5.65 & 0.033 \\
\hline \multicolumn{6}{|l|}{ Pain } \\
\hline PainDETECT score & 20.07 & 8.66 & 16.93 & 8.05 & 0.144 \\
\hline Baseline pain & 7.54 & 1.50 & 5.94 & 2.40 & 0.003 \\
\hline Flare-up pain & 9.38 & 0.80 & 8.85 & 0.95 & 0.025 \\
\hline
\end{tabular}

Abbreviation: PCT, polytrauma clinical triad.

among the PCT group. This may be related to an increased tendency toward developing central sensitization among those patients suffering from a brain-related disorder or neurotrauma, ${ }^{33}$ but this cannot be confirmed in our sample. According to Freyhagen et al (2006), individuals with neuropathic pain show higher ratings of pain intensity and present with more mood and sleep disorders. ${ }^{21}$

Patients with PCT appear to have worse sleep quality, as indicated by higher mean PSQI scores. In a study assessing sleep disturbance in 200 veterans with PCT, Lew et al (2010) reported a high prevalence of sleep disturbance that was attributed to interactions between PTSD and PCS, and those between PTSD and pain. ${ }^{34}$ Worse sleep quality among patients with PCT in our sample is likely attributed to the same underlying mechanisms. Pain and post-traumatic stress following traumatic brain injury can cause prolonged cognitive issues and exacerbate symptoms related to each respective disorder. ${ }^{35,36}$ Further study may provide greater insight into how chronic pain, PTSD, and PCS contribute to the health outcomes of these patients.

In our sample, about half of the patients with chronic pain $(54.9 \%$; $n=39)$ met our modified RPQ-6 scoring criteria for PCS. This more strict approach was limited to visual and vestibular symptoms to prevent misdiagnosis based on symptoms commonly seen in chronic pain and mood disorders. A cutoff score of $\geq 12$ on the RPQ- 6 was used to define PCS in our study population. Because a score of 0 or 1 on any RPQ-6 item indicates "not experienced at all" or "no more of a problem" (than before injury), respectively, an item score of 2 ("a mild problem") or greater is indicative of a symptom that is secondary to injury. ${ }^{37-39}$ Therefore, a PCS screening cutoff score of 12 on the RPQ- 6 was chosen as it relates to an average item score of 2 on each of the six symptoms on the RPQ-6.

A diagnosis of concussion or mTBI was made in fewer than half patients who met screening criteria for PCS (48.7\%; 19 of 39), which is similar to the $42.9 \%$ rate of missed mTBI diagnoses in MVC patients reported by Sharma et al (2014). ${ }^{40}$ These results suggest a potentially high frequency of missed diagnoses of mTBI after MVC, which may lead to worse outcomes in these patients. This may commonly occur among patients presenting with soft tissue pain, in whom a thorough history for possible concussion can be easily overlooked. Factors contributing to missed acute care mTBI diagnosis in these patients include the presence of trauma-associated emotions and issues relating to the treat to life experienced in an MVC, post-traumatic amnesia impairing patient recall of MVC-related events and acute symptoms, subtle neuropathology of mTBI to which neuroimaging (CT scan or conventional MRI) is not sensitive or specific, and an acute care focus on the evaluation and treatment of whiplash-associated pain and injuries. ${ }^{40,41}$ Therefore, it is critical to carefully screen acute MVC patients to rule out possible mTBI. Additionally, it is important to ask patients, first responders, and anyone else present on the scene about confusion, nausea, dizziness, amnesia, and other symptoms that may have resolved before the emergency room assessment takes place.

Using a cutoff score of $\geq 12$ on the modified six-item RPQ, we obtained a PCT prevalence that was similar to that reported in military populations with chronic pain. ${ }^{2}$ This was much more useful than the method of RPQ scoring suggested by Thompson et al, ${ }^{25}$ which yielded an unreasonable $85.9 \%$ prevalence of PCT in our sample (Table 3). These preliminary data suggest that nearly all patients with chronic pain report headaches, mood disturbances, cognitive disturbances, and constitutional symptoms. The remaining six items, which relate to visual and vestibular disturbances, were used to develop a potentially more useful screening tool, the RPQ-6.

Our proposed modified RPQ-6 score should be considered preliminary in nature. This tool was developed based on clinical judgment and is supported by an analysis of patient-reported scores for each item on the RPQ (Table 2). Because our findings support the potential role of a modified RPQ-6 to identify PCS in the presence of chronic pain, further studies should be performed to identify a more reliable clinical tool in this population. Our modified RPQ-6 was a more reliable screening tool for identifying PCS in patients with chronic pain. 
Our study had a number of limitations. While we used validated screening tools for PTSD and PCS during our intake assessments, patients were not formally diagnosed with either syndrome based on an overall clinical impression. As a result, the prevalence of these disorders in our sample was based on symptom scores alone, which may have led to us under-reporting or over-reporting prevalence rates for PCS, PTSD, and consequently PCT. Moving forward, our intake assessment will include a checklist approach that requires the evaluating physician to establish a clinical diagnosis that will be correlated with questionnaire results.

As study measures required patients to retrospectively describe symptom presence, severity, and frequency, variations in patient recall accuracy subject our data to recall bias. ${ }^{42}$ In addition, patients may have felt a need to overreport symptoms for reasons related to secondary gain. They may also have felt a sense of injustice after prolonged unresolved health complaints and disputes with insurers and other providers.

While we believe that our modified RPQ-6 is an important and novel approach to identifying PCS in patients with chronic pain, it has not been prospectively validated, and as such it should be used with caution. However, because the six items measured on the RPQ-6 are drawn from the validated 16-item RPQ, the RPQ-6 was assumed to reliably assess vestibular and visual concussion symptomology and, therefore, accurately screen for PCS. It is more likely that our use of a modified RPQ-6 led us to underreport PCS in our sample.

At present, there are no diagnostic biomarkers for PCS, and its diagnosis is based on the evaluating physician's clinical impression of symptom presence and severity ${ }^{43,44}$ Recent studies have suggested that diffusion tensor imaging abnormalities may correlate to mTBI and PCS incidence. ${ }^{45-48}$ However, its role in clinical care has not yet been established. ${ }^{45}$ Therefore, there is currently no diagnostic tool to compare our findings to and validate the RPQ-6. Future research aiming to validate the RPQ-6 and our proposed cutoff score should be done in comparison to a neuropsychological assessment performed by a neurologist. Screening results can be compared against clinical diagnoses to assess the sensitivity and specificity of the RPQ-6, and to prospectively validate it as a diagnostic instrument for PCS in polytrauma injury patients.

In addition, patient-reported scoring to RPQ- 6 items may become confounded in the presence of chronic pain and other disorders, which could lead to over-reporting PCS prevalence. Dizziness and nausea, two prominent vestibular symptoms associated with PCS, may result from side effects associated with prescription analgesics taken for chronic pain management. ${ }^{49}$ However, less than a quarter of our study population reported current prescription analgesic use (Table 1). Therefore, symptoms of dizziness and nausea reported in our sample are more likely attributed to PCS. Moreover, light sensitivity, a visual symptom associated with PCS, may only present during migraine or migraine-like episodes,$^{50}$ but may be reported by patients as a concussionrelated symptom. Moving forward, our intake assessments will address if RPQ-6-related PCS symptomology is present in the absence of other potential underlying causes.

Our data are subject to selection bias, as our study originates in a population with an established diagnosis of chronic pain. ${ }^{51}$ This may represent a higher-risk population that was referred based on greater perceived impairment. While this selection bias may have led to an increased likelihood of finding a higher prevalence of PCT in our sample, our study is strengthened by the homogeneous nature of the mechanism of injury and subsequent clinical and psychosocial experiences among the study population. While our sample may not be representative of the general chronic pain population, the high prevalence of PCT and the potential role of a modified RPQ-6 as a screening tool in these patients remain notable. Future research should evaluate PCT prevalence in a larger, general sample of patients with chronic pain after MVC.

Our study findings are important because they represent the first report of PCT in a civilian population and support the potentially synergistic effects of chronic pain, PTSD, and PCS in these patients. ${ }^{2,31,32}$ Patients who suffer from PCT may have more difficulty coping with the wide range of symptoms they are experiencing. ${ }^{2}$ This may increase the long-term psychological, social, financial, and health burdens of these disorders and may impair treatment effectiveness. ${ }^{15,16,52}$ Furthermore, the overlapping cognitive and mood disturbance symptoms that are related to chronic pain, PTSD, and PCS $2,18,19,29$ can hinder our ability to accurately attribute the cause of symptoms to a particular disorder. These findings underscore a lack of a "gold standard" in assigning diagnoses when patients present with a myriad of compounding symptomology. However, the high prevalence of PCS reported in this study highlights the need for health care practitioners to accurately screen acute MVC patients to rule out possible mTBI and to consider PCS in patients with persistent whiplash symptoms.

Overall, the results of this study suggest that the PCT is highly prevalent among patients with chronic pain secondary to MVC. In addition, our data support that chronic pain, PCS, and PTSD rarely occur in isolation, as these conditions were found to be more often comorbid with one another. These 
findings highlight the importance of treating the whole person when providing care for these patients, rather than treating a particular disorder. ${ }^{2}$ Additional research is needed to identify the causal factors leading to chronic pain, PTSD, and PCS in these patients, and to better understand the specific factors that lead to the development of PCT. This may lead to earlier and more frequent use of targeted, multidisciplinary assessment and intervention strategies in the future.

\section{Disclosure}

The authors report no conflicts of interest in this work.

\section{References}

1. Pugh MJ, Finley EP, Wang CP, et al. A retrospective cohort study of comorbidity trajectories associated with traumatic brain injury in veterans of the Iraq and Afghanistan wars. Brain Inj. 2016;30(12):1481-1490.

2. Lew HL, Otis JD, Tun C, Kerns RD, Clark ME, Cifu DX. Prevalence of chronic pain, posttraumatic stress disorder, and persistent postconcussive symptoms in OIF/OEF veterans: polytrauma clinical triad. J Rehabil Res Dev. 2009;46(6):697-702.

3. Cifu DX, Taylor BC, Carne WF, Bidelspach D, Sayer NA, Scholten J, Campbell EH. Traumatic brain injury, posttraumatic stress disorder, and pain diagnoses in OIF/OEF/OND veterans. $J$ Rehabil Res Dev. 2013;50(9):1169-1176.

4. Stupar M, Kim PS. Delayed-onset post-traumatic headache after a motor vehicle collision: a case report. J Can Chiropr Assoc. 2007;51(2):83-90.

5. Matar ZS. The clinical profile of poly trauma and management of abdominal trauma in a general hospital in the central region of the kingdom of Saudi Arabia. Internet J Surg. 2008;14:11.

6. Payal P, Sonu G, Anil GK, Prachi V. Management of polytrauma patients in emergency department: an experience of a tertiary care health institution of northern India. World J Emerg Med. 2013;4(1):15-19.

7. U.S. Department of Veteran Affairs. Polytrauma/TBI System of Care. Available from: https://www.polytrauma.va.gov/definitions.asp. Accessed June 5, 2015; cited September 28, 2018.

8. Sullivan KA, Edmed SL. Systematic variation of the severity of motor vehicle accident-related traumatic brain injury vignettes produces different post-concussion symptom reports. Clin Neuropsychol. 2012;26(8):1255-1277.

9. Nash S, Luauté J, Bar JY, et al. Cognitive and behavioural post-traumatic impairments: what is the specificity of a brain injury ? A study within the ESPARR cohort. Ann Phys Rehabil Med. 2014;57(9-10):600-617.

10. Viano DC, Parenteau CS. Concussion, diffuse axonal injury, and AIS4+ head injury in motor vehicle crashes. Traffic Inj Prev. 2015;16(8):747-753.

11. Ursano RJ, Fullerton CS, Epstein RS, et al. Acute and chronic posttraumatic stress disorder in motor vehicle accident victims. Am J Psychiatry. 1999;156(4):589-595.

12. Irish LA, Gabert-Quillen CA, Ciesla JA, Pacella ML, Sledjeski EM, Delahanty DL. An examination of PTSD symptoms as a mediator of the relationship between trauma history characteristics and physical health following a motor vehicle accident. Depress Anxiety. 2013;30(5):475-482.

13. Freeman MD, Croft AC, Rossignol AM, Centeno CJ, Elkins WL. Chronic neck pain and whiplash: a case-control study of the relationship between acute whiplash injuries and chronic neck pain. Pain Res Manag. 2006;11(2):79-83.

14. Jenewein J, Moergeli H, Wittmann L, Büchi S, Kraemer B, Schnyder U. Development of chronic pain following severe accidental injury. Results of a 3-year follow-up study. J Psychosom Res. 2009;66(2):119-126.
15. Humphreys I, Wood RL, Phillips CJ, Macey S. The costs of traumatic brain injury: a literature review. Clinicoecon Outcomes Res. 2013;5:281-287.

16. Mcfarlane AC. The long-term costs of traumatic stress: intertwined physical and psychological consequences. World Psychiatry. 2010;9(1):3-10.

17. Su E, Bell M. Diffuse axonal injury. In: Laskowitz D, Grant G, editors. Translational Research in Traumatic Brain Injury, Chapter 3. Boca Raton (FL): CRC Press/Taylor and Francis Group; 2016. Available from: https://www.ncbi.nlm.nih.gov/books/NBK326722/.

18. Smith-Seemiller L, Fow NR, Kant R, Franzen MD. Presence of postconcussion syndrome symptoms in patients with chronic pain vs mild traumatic brain injury. Brain Inj. 2003;17(3):199-206.

19. Stålnacke BM. Postconcussion symptoms in patients with injury-related chronic pain. Rehabil Res Pract. 2012;2012:Article ID: 528265, 5pp.

20. Treede RD, Rief W, Barke A, et al. A classification of chronic pain for ICD-11. Pain. 2015;156(6):1-1007.

21. Freynhagen R, Baron R, Gockel U, Tölle TR. painDETECT: a new screening questionnaire to identify neuropathic components in patients with back pain. Curr Med Res Opin. 2006;22(10):1911-1920.

22. Weathers FW, Litz BT, Keane TM, Palmieri PA, Marx BP, Schnurr PP. The PTSD Checklist for DSM-5 (PCL-5); Scale available from the National Center for PTSD at www.ptsd.va.gov. Accessed January 23, 2018.

23. Dunn AS, Julian T, Formolo LR, Green BN, Chicoine DR. Preliminary analysis of posttraumatic stress disorder screening within specialty clinic setting for OIF/OEF veterans seeking care for neck or back pain. J Rehabil Res Dev. 2011;48(5):493-502.

24. King NS, Crawford S, Wenden FJ, Moss NE, Wade DT. The Rivermead Post Concussion Symptoms Questionnaire: a measure of symptoms commonly experienced after head injury and its reliability. $J$ Neurol. 1995;242(9):587-592.

25. Thompson C, Paul D, Herrmann L, Summers M, Potter S. Approaches to establishing validated cut-off scores on the Rivermead Post Concussion Symptoms Questionnaire (RPQ). Brain Inj. 2016;30:770.

26. Buysse DJ. Reynolds CF 3rd, Monk TH, Berman SR, Kupfer DJ. The Pittsburgh Sleep Quality Index: a new instrument for psychiatric practice and research. Psychiatry Res. 1989;28(2):193-213.

27. Lagarde E, Salmi LR, Holm LW, et al. Association of symptoms following mild traumatic brain injury with posttraumatic stress disorder vs. postconcussion syndrome. JAMA Psychiatry. 2014;71(9): 1032-1040.

28. Brenner LA, Ivins BJ, Schwab K, Warden D, Nelson LA, Jaffee M, Terrio H. Traumatic brain injury, posttraumatic stress disorder, and postconcussive symptom reporting among troops returning from Iraq. J Head Trauma Rehabil. 2010;25(5):307-312.

29. Bryant R. Post-traumatic stress disorder vs traumatic brain injury. Dialogues Clin Neurosci. 2011;13(3):251-262.

30. Sherin JE, Nemeroff CB. Post-traumatic stress disorder: the neurobiological impact of psychological trauma. Dialogues Clin Neurosci. 2011;13(3):263-278.

31. Otis JD, Keane TM, Kerns RD. An examination of the relationship between chronic pain and post-traumatic stress disorder. J Rehabil Res Dev. 2003;40(5):397-405.

32. Morasco BJ, Lovejoy TI, Lu M, Turk DC, Lewis L, Dobscha SK. The relationship between PTSD and chronic pain: mediating role of coping strategies and depression. Pain. 2013;154(4):609-616.

33. Moshourab RA, Schäfer M, Al-Chaer ED. Chronic pain in neurotrauma: implications on spinal cord and traumatic brain injury. In: Kobeissy FH, editor. Brain Neurotrauma: Molecular, Neuropsychological, and Rehabilitation Aspects, Chapter 11. Boca Raton, FL: CRC Press/Taylor \& Francis; 2015. Available from: https://www.ncbi.nlm.nih.gov/books/ NBK299176/.

34. Lew HL, Pogoda TK, Hsu PT, et al. Impact of the "polytrauma clinical triad" on sleep disturbance in a department of veterans affairs outpatient rehabilitation setting. Am J Phys Med Rehabil. 2010;89(6):437-445. 
35. Combs HL, Berry DT, Pape T, et al. The effects of mild traumatic brain injury, post-traumatic stress disorder, and combined mild traumatic brain injury/post-traumatic stress disorder on returning veterans. J Neurotrauma. 2015;32(13):956-966.

36. Stojanovic MP, Fonda J, Fortier CB, Higgins DM, Rudolph JL, Milberg WP, McGlinchey RE. Influence of mild traumatic brain injury (TBI) and posttraumatic stress disorder (PTSD) on pain intensity levels in OEF/OIF/OND veterans. Pain Med. 2016;17(11):2017-2025.

37. Theadom A, Starkey N, Barker-Collo S, Jones K, Ameratunga S, Feigin V; BIONIC4you Research Group. Population-based cohort study of the impacts of mild traumatic brain injury in adults four years post-injury. PLoS One. 2018;13(1):e0191655.

38. Iverson GL, Lange RT, Wäljas M, et al. Outcome from complicated versus uncomplicated mild traumatic brain injury. Rehabil Res Pract. 2012;2012:415740.

39. Mcmahon P, Hricik A, Yue JK, et al. Symptomatology and functional outcome in mild traumatic brain injury: results from the prospective TRACK-TBI study. J Neurotrauma. 2014;31(1):26-33.

40. Sharma B, Bradbury C, Mikulis D, Green R. Missed diagnosis of traumatic brain injury in patients with traumatic spinal cord injury. J Rehabil Med. 2014;46(4):370-373.

41. Kushner DS. Strategies to avoid a missed diagnosis of co-occurring concussion in post-acute patients having a spinal cord injury. Neural Regen Res. 2015;10(6):859-861.

42. Coughlin SS. Recall bias in epidemiologic studies. J Clin Epidemiol. 1990;43(1):87-91.
43. Boake C, Mccauley SR, Levin HS, et al. Diagnostic criteria for postconcussional syndrome after mild to moderate traumatic brain injury. J Neuropsychiatry Clin Neurosci. 2005;17(3):350-356.

44. Hiploylee C, Dufort PA, Davis HS, et al. Longitudinal study of postconcussion syndrome: not everyone recovers. J Neurotrauma. 2017;34(8):1511-1523.

45. Khong E, Odenwald N, Hashim E, Cusimano MD. Diffusion tensor imaging findings in post-concussion syndrome patients after mild traumatic brain injury: a systematic review. Front Neurol. 2016;7:156.

46. D’Souza MM, Trivedi R, Singh K, et al. Traumatic brain injury and the post-concussion syndrome: a diffusion tensor tractography study. Indian J Radiol Imaging. 2015;25(4):404-414.

47. Zhang J, Wei RL, Peng GP, et al. Correlations between diffusion tensor imaging and levels of consciousness in patients with traumatic brain injury: a systematic review and meta-analysis. Sci Rep. 2017;7(1):2793.

48. Kamnaksh A, Budde MD, Kovesdi E, Long JB, Frank JA, Agoston DV. Diffusion tensor imaging reveals acute subcortical changes after mild blast-induced traumatic brain injury. Sci Rep. 2014;4:4809.

49. Porreca F, Ossipov MH. Nausea and vomiting side effects with opioid analgesics during treatment of chronic pain: mechanisms, implications, and management options. Pain Med. 2009;10(4):654-662.

50. Digre KB, Brennan KC. Shedding light on photophobia. J Neuroophthalmol. 2012;32(1):68-81.

51. Tripepi G, Jager KJ, Dekker FW, Zoccali C. Selection bias and information bias in clinical research. Nephron Clin Pract. 2010;115(2):c94-c99.

52. Phillips CJ. The cost and burden of chronic pain. Rev Pain. 2009;3(1):2-5.
Journal of Pain Research

\section{Publish your work in this journal}

The Journal of Pain Research is an international, peer reviewed, open access, online journal that welcomes laboratory and clinical findings in the fields of pain research and the prevention and management of pain. Original research, reviews, symposium reports, hypothesis formation and commentaries are all considered for publication.

\section{Dovepress}

The manuscript management system is completely online and includes a very quick and fair peer-review system, which is all easy to use. Visit http://www.dovepress.com/testimonials.php to read real quotes from published authors. 